\title{
Correction to: Does Religiosity/Spirituality Play a Role in Function, Pain-Related Beliefs, and Coping in Patients with Chronic Pain? A Systematic Review
}

\author{
Alexandra Ferreira-Valente $\mathrm{e}^{1,2}$ (D) Saurab Sharma ${ }^{3,4} \cdot$ Sandra Torres $^{5,6}$. \\ Zachary Smothers ${ }^{7}$. José Pais-Ribeiro ${ }^{1}$. J. Haxby Abbott ${ }^{3} \cdot$ Mark P. Jensen $^{2}$
}

Published online: 26 October 2019

(c) Springer Science+Business Media, LLC, part of Springer Nature 2019

\section{Correction to: Journal of Religion and Health https://doi.org/10.1007/s10943-019-00914-7}

The original publication of the article contains an error in the co-author name and textual error in Results section.

The family name of the co-author Saurab Sharma should be Sharma instead of Sharmam. The original article has been corrected.

In Results section, last para should read as given below:

The aims we initially proposed in the PROSPERO protocol for this systematic review included examining whether religion is associated with measures of pain and function and the extent to which pain-related beliefs, pain-coping responses and catastrophizing in adults with chronic pain are different or similar in people with different religious affiliation. However, the studies examining such comparisons were

Alexandra Ferreira-Valente and Saurab Sharma have contributed equally to this study.

The original article can be found online at https://doi.org/10.1007/s10943-019-00914-7.

Alexandra Ferreira-Valente

mafvalente@gmail.com

1 William James Center for Research, ISPA - Instituto Universitário, Rua Jardim do Tabaco No 34, 1149-041 Lisbon, Portugal

2 Department of Rehabilitation Medicine, University of Washington, Seattle, USA

3 Centre for Musculoskeletal Outcomes Research, Dunedin School of Medicine, University of Otago, Dunedin, New Zealand

4 Department of Physiotherapy, Kathmandu University School of Medical Sciences, Dhulikhel, Nepal

5 Faculty of Psychology and Education Sciences, University of Porto, Porto, Portugal

6 Centre for Psychology, University of Porto (CPUP), Porto, Portugal

7 Duke University School of Medicine, Durham, USA 
not included in this review, due to concerns about religious affiliation comparisons, noted previously. Twenty studies were included in the final analysis of the systematic review.

Publisher's Note Springer Nature remains neutral with regard to jurisdictional claims in published maps and institutional affiliations. 